Classification

Physics Abstracts

$82.65 \mathrm{D}-68.15-68.45$

\title{
Wetting hydrodynamics
}

\author{
Gary F. Teletzke $\left(^{*}\right)$, H. Ted Davis and L. E. Scriven \\ Department of Chemical Engineering and Materials Science, University of Minnesota, Minneapolis, MN \\ 55455, U.S.A.
}

(Reçu le 28 septembre 1987, révisé le 12 janvier 1988, accepté le 29 février 1988)

\begin{abstract}
Résumé. - Nous généralisons la théorie de Bretherton qui décrit le déplacement stationnaire forcé d'un liquide par un autre dans un tube ou entre plaques parallèles en prenant en compte les effets des forces intermoléculaires dans les films microscopiques et la viscosité finie du fluide qui avance. La théorie prévoit deux régimes d'avancée suivant la valeur du nombre capillaire $\mu U / \gamma$. A faible vitesse, l'angle de contact dynamique apparent garde sa valeur statique. Si le fluide déplacé mouille parfaitement le solide, un film mince existe à l'équilibre. A grande vitesse, un film entraîné de façon hydrodynamique se forme quelles que soient les caractéristiques de mouillage à l'équilibre. L'épaisseur de ce film augmente légèrement avec la viscosité du liquide qui avance. Ces prédictions sont en accord avec l'expérience. De plus, nous montrons que les écarts entre la théorie de Bretherton et les épaisseurs de film mesurées sont dues à la présence d'impuretés tensioactives. Finalement nous discutons les implications sur l'origine microscopique du glissement effectif à la ligne de contact apparente, sur l'entraînement de l'air dans les écoulements de couchage, sur la dynamique des films de savon et sur la génération d'états mixtes de mouillage dans les milieux poreux.
\end{abstract}

\begin{abstract}
Bretherton's (1961) theory of the forced, steady displacement of one fluid by another in a tube or between parallel plates is generalized to account for the effects of intermolecular forces in submicroscopically thin films and of a finite driving-fluid viscosity. The theory predicts that there are two displacement regimes, depending on capillary number $\mu U / \gamma$. At low speed the dynamic apparent contact angle retains its static value. If the displaced fluid perfectly wets the solid an equilibrium thin-film remains; if not, there is not a continuous thin-film. At high speed a hydrodynamically entrained film is left behind regardless of equilibrium wetting characteristics. The entrained film thickens slightly with increasing driving-fluid viscosity. These predictions accord with experiment. In addition, evidence is presented that observed discrepancies between Bretherton's theory and measured film thicknesses are caused by the presence of surface-active impurities. Finally, implications for the submicroscopic origins of seeming slip at apparent contact lines, air entrainment in coating flows, soap-film dynamics, and the generation of mixed-wettability states in porous media are discussed.
\end{abstract}

\section{Introduction.}

The theory of the equilibrium shapes and stability of submicroscopically (less than a few microns thick) thin fluid films is now well developed (Mohanty et al., 1981, 1982 ; Mohanty, 1981 and references therein), but the dynamics of deforming, flowing thin-films has received less attraction. Viscous fluid mechanics augmented by disjoining pressure forces arising from fluid/solid and fluid/fluid intermolecular forces in very thin liquid films has already been applied to understanding soap-film rupture, drop

(*) Now with Exxon Production Research Company, P.O. Box 2189, Houston, TX 77252-2189, U.S.A. coalescence, bubble attachment, and biological membrane mechanics (Vrij, 1966 ; Sheludko, 1968 ; Jain et al., 1979, Ivanov and Jain, 1979; and references therein).

Another class of flows in which thin-films are important is the wetting of solid, which is actually the displacement of one fluid by another and so involves the apparent contact line where fluid, liquid and solid seem to meet. The movement of dynamic contact lines can be classified as either spontaneous or forced. In a companion paper (Teletzke et al., 1987) we analyse the spontaneous, unsteady movement of a straight or circular contact line over a solid surface. De Gennes (1985) and Joanny (1986) also have considered spontaneous wetting. In this paper 
we analyse the forced, steady movement of a dynamic contact line. Joanny and Andelman (1988) have also investigated the steady-state motion of the contact line. The prototype flow considered here is relevant to the displacement of one fluid by another from a small-bore tube or from between closely spaced parallel plates, the coating of solids, and the pulling of a soap film from a liquid bath or Plateau border of a foam when the film surface is inextensible (Fig. 1).

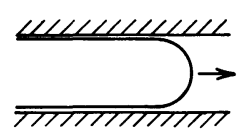

(a)

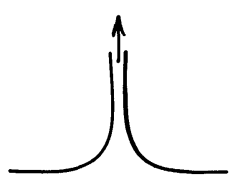

(c)

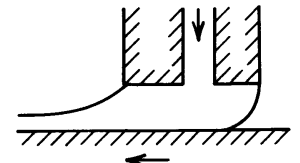

(b)

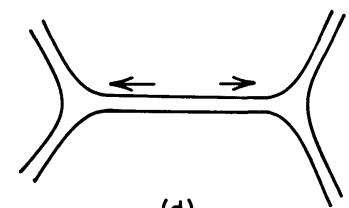

(d) the solid leave films much thicker than predicted by his theory. Bretherton's analysis also implies that the dynamic contact angle is zero regardless of the displacement speed, and thus is unable to describe situations where the liquid is not perfectly wetting.

The drawing of a soap film from a liquid bath has been treated with an analysis analogous to Bretherton's (Mysels, Shinoda and Frankel, 1959 ; Lyklema, Scholten and Mysels, 1965). Again, good agreement between theory and experiment is found except at the slowest displacement speeds, where the film is either much thicker or thinner than predicted by theory, depending on the composition of the film. Likewise, Landau and Levich's (1942) analysis of dip-coating is also analogous to Bretherton's analysis. Levich (1962) reports confirmation of the predicted film thickness, although the range of coating speeds studied is not specified.

These discrepancies between theory and observation are resolved by generalizing Bretherton's analysis in order to account for the effects of intermolecular forces in microscopically thin films. The results provide insight into the physics of flow in the vicinity of the apparent dynamic contact line where a moving meniscus appears to intersect a solid surface. The approach presented here is an alternative to the Navier slip condition introduced by Huh and Scriven (1971) and exploited by many others (Hocking, 1976 ; Huh and Mason, 1977 ; Dussan, 1979 ; Silliman, 1979) to relieve the apparent infinite force singularity at the dynamic contact line.

In addition, Bretherton's analysis is further generalized to account for a viscous rather than an inviscid displacing fluid. Finally, some effects of surface tension gradients on the film deposition process are considered.

\section{Theory of forced displacement.}

A meniscus between two immiscible phases advancing steadily with velocity $U$ through a tube or between parallel plates often leaves behind a film of the fluid which is being displaced (Fig. 2). Unless

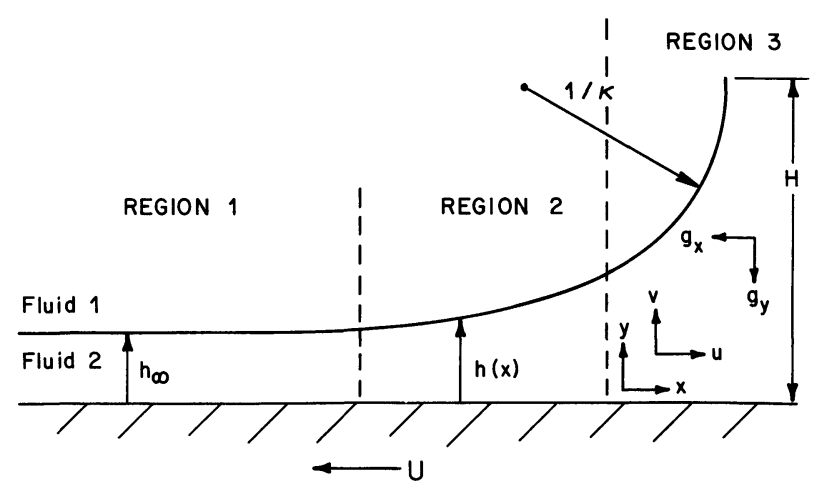

Fig. 2. - Steady displacement between parallel plates or in a tube. slowest displacement speeds. At those speeds Bretherton observed that liquids which perfectly wet 
and until it suffers instability, which may lead to breakup, the film has uniform thickness $h$ which is determined by the balance between capillarity, viscous shear, and disjoining pressure.

The steady, two-dimensional flow configuration is shown in figure 2. Provided the displacement speed measured by the capillary number $N_{\mathrm{Ca}} \equiv U \mu / \gamma_{\mathrm{FF}}$, where $\mu$ is the viscosity of the displaced fluid and $\gamma_{\mathrm{FF}}$ is the tension of the fluid/fluid interface, is not larger than about $10^{-2}$ (Bretherton, 1961), the leading portion of the meniscus, region 3 , is dominated by capillarity and thus is to a good approximation a static meniscus (regardless of the ratio of viscosities of displaced and displacing fluids; see below). Behind is a uniform film in rectilinear flow, region 1 , a regime that is reached asymptotically. Between is the transition region 2 within which the film profile is governed by equations to be derived below.

It is assumed here, as in Bretherton's (1961) analysis, that the Weber number is small, i.e.

$$
\Delta \rho H U^{2} / \gamma_{\mathrm{FF}} \ll 1,
$$

where $H$ is the slot half-width or tube radius, so that inertial effects can be neglected, and that the Bond number is small too, i.e.,

$$
\Delta \rho g H^{2} / \gamma_{\mathrm{FF}} \ll 1,
$$

so that gravitational effects can be neglected.

The equation governing the film profile can be derived by a procedure equivalent to performing a balance of viscous, capillary and disjoining pressure forces and momentum fluxes acting on a slice of film of differential thickness in the $x$-direction (Higgins and Scriven, 1979 : Teletzke, 1983, Teletzke et al., 1987). The appropriate equation is that for the lubrication or low-slope approximation, which is valid provided that where viscous effects are appreciable, the film surface is no more than slightly inclined to the solid surface (Teletzke et al., 1987 ; Teletzke, 1983) :

$$
\begin{aligned}
0= & (H-h) \frac{\partial \gamma_{\mathrm{SF}}}{\partial x}+(H-h) h \frac{\partial}{\partial x}(2 \mathcal{H}) \gamma_{\mathrm{FF}}- \\
& -h\left[\tau_{x y}^{(1)}(x, H)-\tau_{x y}^{(1)}(x, h)\right]+ \\
& +(H-h)\left[\tau_{x y}^{(2)}(x, h)-\tau_{x y}^{(2)}(x, 0)\right]
\end{aligned}
$$

where $\gamma_{\mathrm{SF}}$ is the solid-film tension due to intermolecular forces, $2 \mathcal{H}$ is twice the mean curvature of the fluid-fluid interface, $\gamma_{\mathrm{FF}}$ is the fluid-fluid interfacial tension and $\tau_{x y}$ is the shear component of the viscous stress tensor. The superscripts 1 and 2 denote the displacing and displaced fluids, respectively. In a thick thin-film (i.e., a film thick enough that its fluid/fluid and fluid/solid interfacial zones do not overlap), $\partial \gamma_{\mathrm{SF}} / \partial x$ can be expressed in terms of the disjoining pressure $\Pi$ as (Teletzke et al., 1987) :

$$
\frac{\partial \gamma_{\mathrm{SF}}}{\partial x}=h \frac{\partial \Pi}{\partial h} \frac{\partial h}{\partial x}
$$

How different types of intermolecular forces contribute to the dependence of disjoining pressure on film thickness is reviewed in the next section. Consistent with equation(1), a lubrication type approximation to the velocity profile is appropriate. For now between parallel plates, a semi-parabolic velocity profile,

$$
u(x, y)=F_{0}(x)+y F_{1}(x)+y^{2} F_{2}(x),
$$

is the simplest that satisfies the following requirements : the no-slip boundary condition at the wall (written here in a frame of reference fixed with respect to the moving meniscus),

$$
u_{2}(x, 0)=-U
$$

the requirement of constant flux of each phase in the $x$-direction,

$$
\begin{aligned}
& \int_{0}^{h(x)} u_{2} \mathrm{~d} y=\int_{0}^{h_{\infty}} u_{2} \mathrm{~d} y \equiv q_{2} \\
& \int_{h(x)}^{H} u_{1} \mathrm{~d} y=\int_{h_{\infty}}^{H} u_{1} \mathrm{~d} y \equiv q_{1} ;
\end{aligned}
$$

the condition that flow be symmetric about the midplane of the slot, $y=H$,

$$
\left(\frac{\partial u_{1}}{\partial y}\right)_{H}=0
$$

continuity of velocity at the film surface.

$$
\left(u_{1}\right)_{h}=\left(u_{2}\right)_{h},
$$

and, in low-slope approximation, the condition of continuity of shear stress at the film surface,

$$
\mu_{1}\left(\frac{\partial u_{1}}{\partial y}\right)_{h} \sim \mu_{2}\left(\frac{\partial u_{2}}{\partial y}\right)_{h}
$$

where $\mu_{1}$ is the viscosity of fluid 1 and $\mu_{2}$ is the viscosity of fluid 2 . The velocity profiles constructed to satisfy these conditions are :

$$
u_{1}=C_{1} \frac{(H-v)^{2}-(H-h)^{2}}{2 \mu_{1}}+u_{2}(h),
$$

and

$$
\begin{aligned}
u_{2}=-C_{2} \frac{H^{2}-(H-v)^{2}}{2 \mu_{2}}- & \\
& -\left(C_{1}-C_{2}\right) \frac{(H-v) h}{\mu_{2}}-U,
\end{aligned}
$$

where

$$
C_{1} \equiv \frac{6 U \mu_{1}}{h}\left\{\frac{2 h+3\left(h-h_{\infty}\right)+3 C_{3}}{4(H-h)^{2}+3 m(H-h) h}\right\}
$$




$$
\begin{aligned}
& C_{2} \equiv \frac{6 U \mu_{2}}{h^{3}} \times \\
& \times\left\{\frac{4(H-h)\left(h_{\infty}-h\right)+3 m h(H-h)\left(2 h_{\infty}-h\right)}{4(H-h)^{2}+3 m(H-h) h}-\right. \\
& \left.-C_{3} \frac{2(H-h)^{2}+6 m(H-h) h}{4(H-h)+3 m(H-h) h}\right\} \\
& C_{3} \equiv \frac{m\left(3 H-h_{\infty}\right) h_{\infty}^{2}}{2\left(H-h_{\infty}\right)^{2}+3 m\left[H^{2}-\left(H-h_{\infty}\right)^{2}\right]}
\end{aligned}
$$

and

$$
m \equiv \mu_{1} / \mu_{2} .
$$

Inserting $u_{1}$ and $u_{2}$ into the momentum balance equation (1) yields for the film profile equation in low-slope approximation

$$
\begin{aligned}
& \frac{\mathrm{d}}{\mathrm{d} x}\left[\frac{h_{x x}}{\left(1+h_{x}^{2}\right)^{3 / 2}}\right]=-\frac{H^{2}}{\gamma_{\mathrm{FF}}} \frac{\partial \Pi}{\partial h} h_{x}+ \\
+ & \frac{12 N_{\mathrm{Ca}}\left(h-h_{\infty}\right)}{\left[4(1-h)^{2}+3 m h(1-h)\right] h^{3}} \times \\
\times & \left\{(1-h)^{2}+m \times\right. \\
\times & {\left.\left[\frac{\left(3-h_{\infty}\right)\left[\left(h+h_{\infty}\right)+(3 m-2) h_{\infty} h\right]}{2\left(1-h_{\infty}\right)^{2}+3 m h_{\infty}\left(2-h_{\infty}\right)}-h^{2}\right]\right\}, }
\end{aligned}
$$

where $h$ and $x$ are both measured in units of the slot half-width $H$. The capillary pressure gradient is given by the left side of this equation. It is balanced by the disjoining pressure gradient and net viscous shear stress, which are given by the terms on the right side. As is reviewed in the next section, the disjoining pressure can be modelled as

$$
\Pi(h)=\sum_{n=1}^{4} \frac{A_{n}}{h^{n}}
$$

for many fluid-solid combinations. When this is the case, the first term on the right side of equation (16) becomes :

$$
\begin{aligned}
-\frac{H^{2}}{\gamma_{\mathrm{FF}}} \frac{\partial \Pi}{\partial h} h_{x} & =\sum_{n=1}^{4} \frac{n A_{n}}{\gamma_{\mathrm{FF}} H^{n-1}} \frac{h_{x}}{h^{h+1}}= \\
& =\frac{L_{1} h_{x}}{h^{2}}+\sum_{a=2}^{4}\left(\frac{L_{n}}{H}\right)^{n-1} \frac{h_{x}}{h^{n+1}} .
\end{aligned}
$$

The independent parameters in (16) are the capillary number $N_{\mathrm{Ca}} \equiv \mu U_{2} / \gamma_{\mathrm{FF}}$, which measures the importance of viscous shear relative to capillary pressure, the viscosity ratio $m=\mu_{1} / \mu_{2}$, and a set of parameters $L_{n} / H$, which are the ratios of characteristic lengths, $L_{n}$, of the various types of molecular interactions to the slot half-width, $H$, the last being a measure of the capillary pressure jump across the meniscus in region 3 . Two special cases of the film profile equation are the augmented Young-Laplace equation (Deryagin, 1955, 1957 ; Mohanty, 1981), which expresses balance of capillary and disjoining pressure in thin-films at rest $\left(N_{\mathrm{CA}}=0\right)$; and the Landau-Levich (1942) equation $\left(m=0, A_{n}=0\right)$ which expresses balance between capillary pressure and viscously generated pressure when disjoining pressure gradients and the viscosity of the outer fluid are negligible.

Solutions of the augmented Young-Laplace equation are discussed by Mohanty et al. (1981, 1982) who found the entire equilibrium interfacial profile in the fluid-liquid-solid contact region and from it the equilibrium apparent contact angle. This is the angle at which the visible interfacial profile appears, in the eye's extrapolation, to intersect the solid surface (Fig. 3). From the augmented YoungLaplace equation it is

$\cos \theta_{\mathrm{a}}=1+\left[\Pi\left(h_{\infty}\right) h_{\infty}+\int_{h_{\infty}}^{\infty} \Pi\left(h^{\prime}\right) \mathrm{d} h^{\prime}\right] / \gamma_{\mathrm{FF}}$

which can be shown to be identical to Young's equation for the apparent contact angle (Teletzke, 1983). The apparent contact angle under dynamic conditions is examined below.

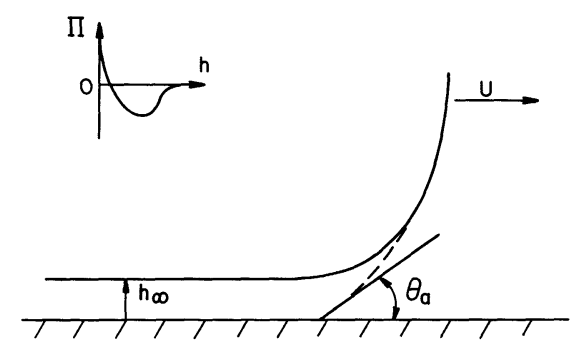

Fig. 3. - Illustration of apparent contact angle.

The Landau-Levich equation was originally used to predict the thickness of the film deposited on a solid substrate being withdrawn vertically from a liquid bath. In that case it is appropriate to measure film thicknesses in units of the capillary length $\equiv(\gamma / 2 \rho g)^{1 / 2}$ rather than the slot half-width. When a surfactant is present that renders the liquid-vapor interface inextensible in the transition region, the same equation can be used to predict the thickness of a soap-film drawn vertically from a bath (Mysels et al., 1959 ; Lyklema et al., 1965). Furthermore, the Landau-Levich equation is identical to that used by Bretherton (1961) in his analysis of the motion of long bubbles in tubes.

Bretherton's work established that when disjoining pressure is negligible, the next higher-order terms neglected from equation(16), which are pro- 
portional to $N_{\mathrm{Ca}} h_{x}^{2}$ or $N_{\mathrm{CA}} h h_{x x}$, are smaller than the terms included in equation (16) by $O\left(N_{\mathrm{Ca}}^{2 / 3}\right)$ in region 2 . In such cases the small-slope approximation leads to self-consistent results for $N_{\mathrm{Ca}} \ll 1$. When the disjoining pressure function is such that it gives zero apparent contact angle, the calculations presented below confirm the self-consistency of the small-slope approximation for $N_{\mathrm{Ca}} \ll 1$. However, when the disjoining pressure function is such that it gives a large apparent contact angle, the calculations show that the film profile may have neither small slope nor curvature in region 2 , and that therefore the smallslope approximation fails.

A completely analogous procedure leads to the equation describing the film profile in a tube (Teletzke, 1983); the equation is lengthy and only the special case for $m=0$ is given here :

$$
\begin{aligned}
\frac{\mathrm{d}}{\mathrm{d} x}\left[\frac{r_{x x}}{\left(1+r_{x}^{2}\right)^{3 / 2}}\right. & \left.-\frac{1}{r\left(1+r_{x}^{2}\right)^{1 / 2}}\right]=\frac{H^{2}}{\sigma_{\mathrm{FF}}} \frac{\partial \Pi}{\partial r} \Gamma_{x}+ \\
& +\frac{8 N_{\mathrm{Ca}}\left(r^{2}-r^{2}\right)}{\left[1-4 r^{2}+3 r^{4}-4 r^{4} \ln r\right]},
\end{aligned}
$$

where $H$ is the tube radius, and $r \equiv 1-h / H$, where $h$ is film thickness. If $h / H \ll 1$ everywhere, (20) becomes

$$
\begin{aligned}
\frac{\mathrm{d}}{\mathrm{d} x}\left[\frac{h_{x x}}{\left(1+h x^{2}\right)^{3 / 2}}\right]=\frac{H^{2}}{\sigma_{\mathrm{FF}}} & \frac{\partial \Pi}{\partial h} h_{x}+ \\
& +\frac{3 N_{\mathrm{Ca}}\left(h-h_{\infty}\right)}{h^{3}},
\end{aligned}
$$

which is the same as (16) for $m=0$. This shows that if $h \ll 1$, the profile of the film in a tube is identical to the profile of the film between two plates having gap width $H$ equal to the tube diameter, a result confirmed by the calculations to be discussed later.

\section{Disjoining pressure functions.}

It is customary to divide disjoining pressure into three contributions : molecular, $\Pi_{\mathrm{m}}$, electrostatic, $\Pi_{\mathrm{e}}$, and structural, $\Pi_{\mathrm{s}}$. To calculate how $\Pi_{\mathrm{m}}$ and $\Pi_{\mathrm{e}}$ depend on film thickness it is usually assumed that the film, whether it be liquid or vapor, has uniform density equal to that of saturated bulk fluid, i.e. fluid in equilibrium with a second bulk fluid phase (Sheludko, 1968 ; Buscall and Ottewill, 1975). A consequence of this assumption is that disjoining pressure is the negative derivative with respect to film thickness of the interaction energy, $\omega \equiv f_{\mathrm{f}}-f_{\mathrm{b}}$, $f_{\mathrm{f}}$ being the Helmholtz free energy per unit area of the film, and $f_{\mathrm{b}}$ that of bulk fluid (Deryagin and Kussakov, 1937, 1939) :

$$
\begin{aligned}
\Pi \equiv\left(\rho^{(2)}-\rho^{(1)}\right)[\mu(h, T) & -\mu(h=\infty, T)]= \\
& =-\left(\frac{\partial \omega}{\partial h}\right)_{T, V, A}
\end{aligned}
$$

where $\rho^{(2)}$ is the density of the film and $\rho^{(1)}$ that of bulk fluid. To calculate $\Pi_{\mathrm{m}}$ it is usually further assumed that entropic contributions to the Helmholtz free energy are negligible, i.e., the Helmholtz free energy equals the internal energy. This latter approximation is accurate for thick thin-films, but most certainly breaks down for thin thin-films, in which the structural contribution $\Pi_{\mathrm{s}}$ predominates (Teletzke et al., 1982).

Calculation of the molecular contribution to disjoining pressure $\Pi_{\mathrm{m}}$ has been approached in two ways : from the approximation of interactions as pairwise additive, and from a field theory of manybody interactions in condensed matter. The simpler and historically earlier approach is a theory based on summing individual London-Van der Waals interactions between molecules pair-by-pair (DeBoer, 1936 ; Hamaker, 1937)), supplemented later with the correction for finite propagation speed of the responsible electromagnetic waves (Casimir and Polder, 1948). The more sophisticated, modern theory of $\Pi_{\mathrm{m}}$ was developed by Lifshitz and coworkers $(1956,1960)$, who took the interactions among the film and its two surrounding phases to be through the intermediary of a fluctuating electromagnetic field. The frequency-dependent dielectric constant $\varepsilon$ of the film is approximated by the subscript 1 , between two semi-infinite phases, 2 and 3 ; the disjoining pressure, $\Pi_{\mathrm{m}}$, is given by (Lifshitz, 1956)

$$
\begin{aligned}
\Pi_{\mathrm{m}}=\frac{\hbar}{2 \pi^{2} c^{3}} \int_{0}^{\infty} \int_{1}^{\infty} p^{2} \xi^{3} \varepsilon_{1}^{3 / 2}\{[ & {\left[\frac{\left(s_{2}+p\right)\left(s_{3}+p\right)}{\left(s_{2}-p\right)\left(s_{3}-p\right)} \exp \left(\frac{2 p \xi h \sqrt{\varepsilon_{1}}}{c}\right)-1\right]+} \\
& \left.+\left[\frac{\left(s_{3}+p \varepsilon_{3} / \varepsilon_{1}\right)\left(s_{2}+p \varepsilon_{2} / \varepsilon_{1}\right)}{\left(s_{3}-p \varepsilon_{3} / \varepsilon_{1}\right)\left(s_{2}-p \varepsilon_{2} / \varepsilon_{1}\right)} \exp \left(\frac{2 p h \sqrt{\varepsilon_{1}}}{c}\right)-1\right]^{-1}\right\} \mathrm{d} p \mathrm{~d} \xi
\end{aligned}
$$

where $\pi \hbar \equiv$ Plancks constant, $c \equiv$ speed of light, $s_{2} \equiv\left(\varepsilon_{2} / \varepsilon_{1}-1+p^{2}\right)^{1 / 2}, s_{3} \equiv\left(\varepsilon_{3} / \varepsilon_{1}-1+p^{2}\right)^{1 / 2}$, and the dielectric constants $\varepsilon_{1}, \varepsilon_{2}$ and $\varepsilon_{3}$ are functions of imaginary frequency $\omega \equiv i \xi$ and are given by

$$
\varepsilon(i \xi)=1+\frac{2}{\pi} \int_{0}^{\infty} \frac{\omega \varepsilon^{\prime \prime}(\omega)}{\omega^{2}+\xi^{2}} \mathrm{~d} \omega,
$$


where $\varepsilon^{\prime \prime}(\omega)$ is the imaginary component of the dielectric constant. $\varepsilon^{\prime \prime}(\omega)$ corresponds to the dissipation to heat of the electromagnetic wave and hence is always positive.

In the limiting case of film thickness $h$ small in comparison with the characteristic wavelengths $\lambda_{0}$ of the adsorption spectra of the bodies concerned, Lifshitz (1956) showed that disjoining pressure is inversely proportional to the cube of film thickness. Equation (23) reduces to

$$
\Pi_{\mathrm{m}}=\frac{-\hbar}{16 \pi^{2} h^{3}} \int_{0}^{\infty} \int_{0}^{\infty} x^{2}\left[\frac{\left(\varepsilon_{1}+\varepsilon_{3}\right)\left(\varepsilon_{2}+\varepsilon_{3}\right)}{\left(\varepsilon_{3}-\varepsilon_{1}\right)\left(\varepsilon_{2}-\varepsilon_{3}\right)} e^{x}-\right] \mathrm{d} x \mathrm{~d} \xi
$$

In the limiting case of $\mathrm{h}$ large in comparison to $\lambda_{0}$, on the other hand, disjoining pressure turns out to be inversely proportional to the fourth power of film thickness :

$$
\begin{aligned}
& \Pi_{\mathrm{m}}=\frac{-\hbar}{32 \pi^{2} h^{4} \sqrt{\varepsilon_{10}}} \times \\
& \int_{0}^{\infty} \int_{0}^{\infty} \frac{x^{2}}{p^{2}}\left\{\left[\frac{\left(s_{30}+p\right)\left(s_{20}+p\right)}{\left(s_{30}-p\right)\left(s_{20}-p\right)} \mathrm{e}^{x}-1\right]^{-1}+\left[\frac{\left(s_{30}+p \varepsilon_{30} / \varepsilon_{10}\right)\left(s_{20}+p \varepsilon_{20} / \varepsilon_{10}\right)}{\left(s_{30}-p \varepsilon_{30} / \varepsilon_{10}\right)\left(s_{20}-p \varepsilon_{20} / \varepsilon_{10}\right)} \mathrm{e}^{x}-1\right]^{-1}\right\} \mathrm{d} p \mathrm{~d} x,
\end{aligned}
$$

where $s_{30} \equiv\left(\varepsilon_{30} / \varepsilon_{10}-1+p^{2}\right)^{1 / 2}, s_{20} \equiv\left(\varepsilon_{30} / \varepsilon_{10}-1+p^{1 / 2}\right)^{1 / 2} . \varepsilon_{10}, \varepsilon_{20}$ and $\varepsilon_{30}$ and $\varepsilon_{30}$ are the electrostatic values of the dielectric constant at $\xi=0$.

Until a decade ago it was believed that the Lifshitz theory of Van der Waals forces was but an elegant formalism, since the necessary dielectric constants across the entire frequency range could not readily be determined. Then Parsegian and Ninham (1969) discovered a technique for calculating these properties to an adequate approximation from dielectric data available in handbooks (Mahanty and Ninham, 1976). Precise measurements of $\Pi_{\mathrm{m}}$ in thick thinfilms, as reviewed by Mahanty and Ninham (1976), are in good agreement with Lifshitz theory. Lifshitz theory does not apply however to films so thin as to have dielectric properties which vary with thickness.

The molecular or Van der Waals contribution, $\Pi_{\mathrm{m}}$, represents an unstructured thin-film in the sense that no entropy component of free energy is accounted for. But when ionic or polar species are present in a liquid, electrostatic interactions can order those species over distances of $10^{-5} \mathrm{~cm}$ or even $10^{-4} \mathrm{~cm}$ near each surface of a thick thin-film. The ordering is opposed by thermal motion. The net result is the so-called diffuse electric double layer (Verwey and Overbeek, 1948 ; Deryagin and Land$\mathrm{au}, 1941)$. The two double layers, one at each surface of a thin-film, come into interference and compress each other; the result is an extra pressure. $\Pi_{\mathrm{e}}$, which is the ionic-electrostatic contribution to disjoining pressure. Estimates have been calculated by Langmuir (1938), Frankel (1946), Verwey and Overbeek (1948), Deryagin and Landau (1941), and Sheludko (1968) for two interfaces at the same constant surface potential $\psi_{0}$ in a symmetrical electrolyte of valence $z$, i.e., a binary electrolyte in which the valence of one component is $z$ and the valence of the other component is $-z$ :

$$
\Pi_{\mathrm{e}}=2 \rho_{\mathrm{i}} k T[\cosh u-1] \text {. }
$$

Here $\rho_{\mathrm{i}}$ is the ion number density in the bulk solution and $u \equiv z e \psi_{\mathrm{d}} / k T ; e$ is the elementary electronic charge, and $\psi_{\mathrm{d}}$ is the electrostatic potential midway between the two surfaces. The thickness, $h$, of a film under these conditions is (Verwey and Overbeek, 1948)

$$
h=-\frac{2}{\chi} \int_{u_{0}}^{u} \frac{\mathrm{d} y}{2[\cosh y-\cosh u]^{1 / 2}},
$$

where $u_{0} \equiv z e \psi_{0} / k T$ and $y \equiv z e \psi / k T . \quad \chi$ is the reciprocal of the screening distance (often referred to as the Debye length) of the double layer and for a symmetrical electrolyte is given by

$$
\chi=\left(8 \pi \rho_{\mathrm{i}} z^{2} e^{2} / \varepsilon k T\right)^{1 / 2}
$$

$\varepsilon$ is the dielectric constant of fluid constituting the film. The integral in (28) was tabulated by Verwey and Overbeek (1948). For $\chi h<2, \Pi_{\mathrm{e}}$ can be approximated by

$$
\Pi_{\mathrm{e}}=64 n k T \tanh ^{2}\left(\frac{u_{0}}{2}\right) \exp (-\chi h) .
$$

Therefore, when the potential is symmetric $\cosh u>1$ and $\Pi_{\mathrm{e}}>0$. This is the case in a liquid film between two identical fluids or solids.

In cases of liquid films between a solid and another fluid, the electrostatic potentials in general differ at the two interfaces. If the values are $\psi_{1}$ and $\psi_{2}$, respectively, then for $\chi h>2$ (Hogg, Healy and Fuerstenau, 1966)

$$
\begin{aligned}
\Pi_{\mathrm{e}}=\frac{\varepsilon \chi^{2}}{8 \pi}\{ & \left(\psi_{1}^{2}+\psi_{2}^{2}\right)\left[1-\operatorname{coth}^{2}(\chi h)\right]- \\
& \left.-2 \psi_{1} \psi_{2} \operatorname{csch}(\chi h) \operatorname{coth}(\chi h)\right\}
\end{aligned}
$$


If the potential vanishes on one interface,

$$
\Pi_{\mathrm{e}}=\frac{e \chi^{2}}{8 \pi} \psi^{2}\left[1-\operatorname{coth}^{2}(\chi h)\right] .
$$

This is negative; hence $\Pi_{\mathrm{e}}$ can be positive or negative depending on the surface potentials.

An important result is that $\Pi_{\mathrm{e}}$ decreases with increasing electrolyte concentration at fixed surface potential and film thickness, according to equations (29) and (30) or (31). When both surfaces of the film have a high potential and the concentration of symmetrical electrolyte is low, disjoining pressure is inversely proportional to the square of the film thickness (Deryagin and Landau, 1941):

$$
\Pi_{\mathrm{e}}=\frac{\pi \varepsilon}{8}\left(\frac{k T}{e z}\right)^{2} \frac{1}{h^{2}} .
$$

This relation is verified by experimental measurements on water films on glass, quartz and mica (Deryagin and Churaev, 1974).

The case of nonsymmetrical electrolytes is described by a simple modification of the foregoing (Deryagin and Landau, 1941). Devereaux and de Bruyn (1963) give details and numerical results. The effect of constant charge rather than constant potential at the interfaces is considered by Ohshima (1974). The effect of liquid structure in the first molecular layer or so from the solid, the so-called Stern Layer, and the interaction of $\Pi_{\mathrm{e}}$ with the other contributions to disjoining pressure is not yet known and needs to be studied.

The structural contribution, $\Pi_{s}$, of disjoining pressure covers the modification of structure induced in a thin thin-film by surface forces not accounted by $\Pi_{\mathrm{m}}$ and $\Pi_{\mathrm{e}}$. This contribution represents the entropic contribution to chemical potential (or free energy) in a thin layer due to orientation, solvation and other specific interactions (Deryagin and Churaev, 1974).

In all fluids steric interaction is short-ranged and hence $\Pi_{s}$ is important for thin thin-films at least. Teletzke, Scriven and Davis (1982) investigated with the gradient theory of inhomogeneous fluid the structural component of disjoining pressure in films of simple nonpolar molecules. Deviations from the inverse cubic dependence on film thickness in films thinner than 10-15 molecules were found in rough agreement with experiment (Deryagin and Churaev, 1974). The structural component can, however, be important in much thicker films. Pashley (1980) reported that water films on clean quartz have a structural component, $\Pi_{\mathrm{s}}$, very large compared to the molecular or ionic or electrostatic component at thicknesses less than $80 \mathrm{~nm}$. He established that in this range $\Pi_{\mathrm{s}}$ varies inversely with film thickness, i.e., $\Pi_{\mathrm{s}} \propto h^{-1}$. A theoretical explanation for this large structural component in water is presently lacking but it is suspected to be due primarily to hydrogen bonding. On contaminated or dehydroxylated quartz, however, it was found that the structural component changes sign at a film thickness of about $10 \mathrm{~nm}$ (Pashley, 1980).

At high enough electrolyte concentration in aqueous solution, short-range repulsive hydration forces, apparently caused by the interaction of hydrated counterions adsorbed on the solid-liquid or liquid-fluid interface have been observed. (Pashley, 1981 ; Pashley and Quirk, 1984). The contribution of hydration forces to disjoining pressure can be modelled as an exponential dependence on film thickness (Pashley and Quirk, 1984), which depends strongly on the valence and size of the adsorbed counterions.

The nonadditive interactions between individual contributions are not fully known and so the total disjoining pressure in a thin film is approximated as the sum of all the above-named contributions. This has never been critically tested. Each contribution can be positive or negative depending on the constitution of the three phases involved - the solid, liquid and fluid - and so disjoining pressure can vary with thickness and change sign in a variety of ways. Figure 4 lists representative possibilities catalogued by Dzyaloshinskii, Lifshitz and Pitaevskii (1960). The functional form of disjoining pressure governs equilibrium film states and their stability (Mohanty, 1981 ; Mohanty et al, 1981, 1982) as well as the flow of nonequilibrium films, as shown below. It is only when $\mathrm{d} \Pi / \mathrm{d} h<0$ that disjoining pressure truly disjoins the two surfaces of a thin-film. For this reason the more descriptive term conjoining pressure will be used henceforth in situations where $\mathrm{d} \Pi / \mathrm{d} h>0$.

(a)

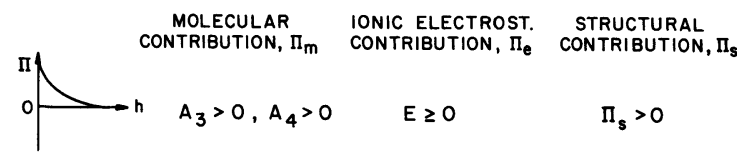

(b)

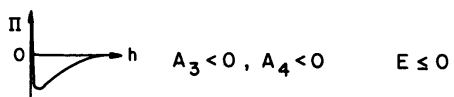

$\Pi_{s}>0$

(c)

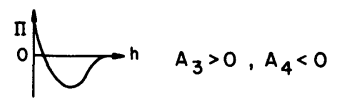

$E \leq 0$

$\Pi_{s}>0$

(d)

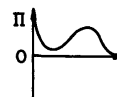

$A_{3}<0, A_{4}>0 \quad E \geq 0$

$\Pi_{s}>0$

(e)

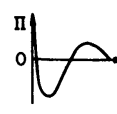

$A_{3}<0, A_{4}>0 \quad E \geq 0$

$\Pi_{s}>0$

Fig. 4. - Disjoining pressure functions.

Not uncommonly it turns out that just one of the contributions to disjoining pressure dominates all the others. In aqueous films on quartz the ionicelectrostatic component, $\Pi_{\mathrm{e}}$, predominates at film thicknesses greater than $h>150 \mathrm{~nm}$ and the struc- 
Table I. - Contributions to disjoining pressure.

\begin{tabular}{|c|c|c|}
\hline Contribution & Origin & Approximate \\
\hline Molecular $\Pi_{\mathrm{m}}$ & $\begin{array}{l}\text { London Van der Waals dispersion } \\
\text { including electromagnetic retardation }\end{array}$ & $\begin{array}{l}\text { Dependence on } h \\
A_{3} / h^{3}, \text { small } h \\
A_{4} / h^{4}, \text { large } h\end{array}$ \\
\hline Ionic-Electrostatic $\Pi_{\mathrm{e}}$ & $\begin{array}{l}\text { Overlapping of double layers } \\
\text { Surface charge-dipole interaction }\end{array}$ & $\begin{array}{l}E \mathrm{e}^{-\chi^{h}}, \chi h \text { large } \\
A_{2} / h^{2}, \text { pure water }\end{array}$ \\
\hline Structural $\Pi_{\mathrm{s}}$ & $\begin{array}{l}\text { Short range forces } \\
\text { Hydrogen bonding } \\
\text { Hydration forces }\end{array}$ & $\begin{array}{l}A_{0} \ln h \\
A_{1} / h \text { water on quartz } \\
A_{\mathrm{H}} \mathrm{e}^{-h / D} \text { aqueous electrolyte on mica }\end{array}$ \\
\hline
\end{tabular}

tural component $\Pi_{\mathrm{s}}$ predominates at $h<80 \mathrm{~nm}$ (Pashley, 1980). With decane on metals (Deryagin and Churaev, 1974) the fully retarded limit of the molecular component $\left(\Pi \sim A_{4} / h^{4}\right)$ is a good approximation if $h>40 \mathrm{~nm}$ whereas the unretarded limit of the molecular component is adequate for $2 \mathrm{~nm}<h<10 \mathrm{~nm}$. The exact demarcation between different regimes depends on the particular set of phases involved. Table I summarizes the different contributions and lists their approximate dependence on thickness.

\section{Method of solution.}

Equation (16) is a third-order ordinary differential equation elliptic in character because of the capillary pressure gradient, which transmits information upstream. The problem is similar to a two-point boundary value problem, except that definite boundaries are lacking: there are only asymptotic conditions upstream and downstream. The upstream asymptotic condition is that meniscus curvature tends to a constant value, $\kappa$, near the centerline of the slot,

$$
\frac{h_{x x}}{\left(1+h_{x}^{2}\right)^{3 / 2}} \rightarrow \kappa \quad \text { as } \quad h \rightarrow 1 .
$$

A cylindrical meniscus is uniquely specified by its curvature $\kappa$ and by the location of its center $\left(x_{0}, y_{0}\right)$. If the static meniscus is centered in the slot, i.e., $y_{0}=H$, then the candidate static menisci belong to a two-parameter family of solutions, the parameters being $x_{0}$ and $\kappa$. The downstream asymptotic condition is that the film thickness approaches a uniform value, $h_{\infty}$ far downstream of region 3 :

$$
\left.\begin{array}{l}
h \rightarrow h_{\infty} \\
h_{x} \rightarrow 0
\end{array}\right\} \quad \text { as } \quad x \rightarrow-\infty .
$$

The film profile equation is solved by replacing the static meniscus boundary condition with an assign- ment of the residual film thickness, which transforms the boundary value problem into an initial value problem easily solved by numerical integration (Kheshgi and Scriven, 1979; Kistler and Scriven, 1979). For the initial value problem to be wellposed, two more starting conditions are needed. These are obtained from the asymptotic solution of the film profile equation for the approach of the profile to a uniform film.

For small deviations of the film thickness from its asymptotic value $h_{\infty}$ the film profile can be expressed as

$$
h=h_{\infty}+\varepsilon h_{1}(x)+O\left(\varepsilon^{2}\right) .
$$

where $\varepsilon$ is a small constant. By inserting (36) into the film profile equation (16) and equating the coefficient of $\varepsilon$ to zero, a linear, third-order ordinary differential equation with constant coefficients for $h_{1}(x)$ is obtained,

$$
\begin{aligned}
& h_{1 x x x}+\left[\frac{L_{1}}{h_{\infty}^{2}}+\sum_{n=2}^{4}\left(\frac{L_{n}}{H}\right)^{n-1} \frac{1}{h_{\infty}^{n+1}}\right] h_{1 x}- \\
& -\frac{12 N_{\mathrm{Ca}}}{\left[4\left(1-h_{\infty}\right)^{2}+3 m h_{\infty}\left(1-h_{\infty}\right)\right] h_{\infty}^{3}} \\
& \times\left[\frac{\left(3-h_{\infty}\right)\left(2 h_{\infty}+(3 m-2) h_{\infty}^{2}\right)}{2-\left(1-h_{\infty}\right)^{2}+3 m h_{\infty}\left(2-h_{\infty}\right)}-h_{\infty}^{2}\right] h_{1}=0
\end{aligned}
$$

or

$$
h_{1 x x x}+p h_{1 x}+q h_{1}=0 .
$$

The solution of (37) is

$$
h_{1}=L \mathrm{e}^{\gamma_{1} x}+M \mathrm{e}^{\gamma_{2} x}+N \mathrm{e}^{\gamma_{3} x}
$$

where the $\gamma_{k}$ are roots of the indicial equation

$$
\gamma_{k}^{3}+p \gamma_{k}+q=0
$$

If the discriminant $R \equiv(p / 3)^{3}+(q / 2)^{2}>0$, then 
there are one real root and two conjugate complex roots, given by

$$
\begin{aligned}
& \gamma_{1}= {[-q / 2+\sqrt{R}]^{1 / 3}+[-q / 2-\sqrt{R}]^{1 / 3} } \\
& \gamma_{2}=-\frac{1}{2} \gamma_{1}+i \sqrt{3 / 2} \times \\
& \quad \times\left([-q / 2+\sqrt{R}]^{1 / 3}-[-q / 2-\sqrt{R}]^{1 / 3}\right) \\
& \gamma_{3}=-\frac{1}{2} \gamma_{1}-i \sqrt{3 / 2} \times \\
& \quad \times\left([-q / 2+\sqrt{R}]^{1 / 3}-[-q / 2-\sqrt{R}]^{1 / 3}\right) . \quad(41)
\end{aligned}
$$

Because $q<0$, the real root $\gamma_{1}$ is positive and the real parts of $\gamma_{2}$ and $\gamma_{3}$ are negative. If $R<0$, then there are three real roots given by

$$
\begin{array}{r}
\gamma_{k}=(-p / 3)^{1 / 2} \cos \left[(\phi / 3)+\frac{2 \pi}{3}(k-1)\right] \\
k=1,2,3
\end{array}
$$

when $q<0$, where

$$
\phi=\cos ^{-1}\left[\frac{q^{2} / 4}{-p^{3} / 27}\right]^{1 / 2} .
$$

Again, because $q<0$, only one of the roots, $\gamma_{1}$, is positive. Therefore the coefficients $M$ and $N$ in (40) must be set equal to zero to satisfy the downstream asymptotic conditions (35).

If the downstream and upstream asymptotic conditions are interchanged, corresponding to the advance of a meniscus over a previously deposited film, the coefficient $L$ in (39) must be set equal to zero to satisfy the upstream asymptotic conditions

$$
\left.\begin{array}{l}
h \rightarrow h_{\infty} \\
h_{x} \rightarrow 0
\end{array}\right\} \quad \text { as } \quad x \rightarrow \infty .
$$

This situation is discussed elsewhere (Teletzke, 1983 ; Starov, 1977).

Thus the asymptotic solution for the approach of the profile to a uniform film is

$$
h=h_{\infty}+\varepsilon L \mathrm{e}^{\gamma_{1} x} .
$$

This equation describes a two-parameter family of solutions for a given choice of $N_{\mathrm{Ca}}$ and disjoining pressure function, the parameters being $h_{\infty}$ and $\varepsilon L$. Because the exponential growth factor $\gamma_{1}$ depends on $h_{\infty}$ alone, different choices of $\varepsilon L$ at fixed $h_{\infty}$ yield profiles having the same shape, but shifted laterally in the slot.

Three initial conditions for the initial value problem are obtained by evaluating at some arbitrary point $x^{*}$ the expressions for the thickness, slope, and curvature of the asymptotic profile given by (45):

$$
\begin{aligned}
& h=h_{\infty}+\varepsilon L \mathrm{e}^{\gamma_{1} x^{*}}, \\
& h_{x}=\gamma_{1} \varepsilon L \mathrm{e}^{\gamma_{1} x^{*}}, \\
& h_{x x}=\gamma_{1}^{2} \varepsilon L \mathrm{e}^{\gamma_{1} x^{*}} .
\end{aligned}
$$

With the initial conditions specified, numerical integration of the full nonlinear profile equation can begin. The integration proceeds stepwise in the positive $x$ direction using arc length as the coordinate. In terms of arc length $s$, the film profile equation becomes a system of four nonlinear firstorder ordinary differential equations :

$$
\begin{aligned}
\frac{\mathrm{d} x}{\mathrm{~d} s} & =\cos \theta, \\
\frac{\mathrm{d} h}{\mathrm{~d} s} & =\sin \theta, \\
\frac{\mathrm{d} \theta}{\mathrm{d} s} & =(2 \mathscr{H}) \\
\frac{\mathrm{d}}{\mathrm{d} s}(2 H)= & \sin \theta G_{1}(h)+\cos \theta G_{2}(h),
\end{aligned}
$$

where

$$
\begin{gathered}
G_{1}(h) \equiv-\frac{H^{2}}{\gamma_{\mathrm{FF}}} \frac{\partial \Pi}{\partial h} \\
G_{2}(h) \equiv \frac{12 N_{\mathrm{Ca}}\left(h-h_{\infty}\right)}{\left[4(1-h)^{2}+3 m h(1-h)\right] h^{3}}\left\{(1-h)^{2}+\right. \\
\left.m\left[\frac{\left(3-h_{\infty}\right)\left(h+h_{\infty}\right)+(3 m-2) h_{\infty} h}{2\left(1-h_{\infty}\right)^{2}+3 m h_{\infty}\left(2-h_{\infty}\right)}-h^{2}\right]\right\} .
\end{gathered}
$$

$\theta$ is the inclination angle of the local tangent with respect to the solid surface. The initial conditions obtained by expressing (46-48) in terms of arc length are that at $x=x^{*}$ :

$$
\begin{gathered}
h=h_{\infty}+\varepsilon L \mathrm{e}^{\gamma_{1} x^{*}} \\
\theta=\tan ^{-1}\left(h_{x}\right)=\tan ^{-1}\left(\gamma_{1} \varepsilon L \mathrm{e}^{\gamma_{1} x^{*}}\right) \\
2 \mathcal{H}=h_{x x}=\gamma_{1}^{2} \varepsilon L \mathrm{e}^{\gamma_{1} x^{*}} .
\end{gathered}
$$

The set of equations (48-52) is integrated upstream through the developing flow region by means of an implicit integration scheme developed by Hindmarsh (1974). At each step the trajectory is tested to see when viscous and disjoining effects become negligible and meniscus curvature is therefore constant. If $h_{x}$ is small and positive where the static meniscus is first reached, then the approximations leading to the film profile equation are justified. A given assignment of $h_{\infty}$ corresponds to a unique value of the curvature of the static meniscus $\kappa$, and therefore by changing the initial assignment of $h_{\infty}$ different static menisci are found. Changing $\varepsilon L$ changes only the location of the center of the static meniscus, $x_{0}$, but not its curvature. The solution sought is the one having a static meniscus centered in the slot. This is found by adjusting $h_{\infty}$ until the value of meniscus curvature $\kappa$ is found that gives $\theta=\pi / 2$ when $h=1$.

For values of $N_{\mathrm{Ca}}$ larger than about $5 \times 10^{-3}$, 
meniscus curvature does not approach a constant near the center of the slot; this indicates failure of the small-slope approximation. At $N_{\mathrm{Ca}}=5 \times 10^{-3}$, film thickness is four percent of the half slot width. Hence the residual film thickness in a tube is the same as that over the entire range of validity of (16) and (20), $N_{\mathrm{Ca}}<5 \times 10^{-3}$, in a slot having gap halfwidth equal to the tube radius.

\section{Solutions.}

This analysis reveals the dependence of residual film thickness, $h_{\infty}$, on fluid properties, wetting properties as determined by disjoining pressure, slot half-width (or tube radius), and displacement velocity. Shown in figures 5-10 are the results for several types of disjoining pressure functions when inertial and gravitational effects are negligible. Film thickness measured in units of the slot half-width (or tube radius) is plotted against capillary number, $N_{\mathrm{Ca}}$, which is the displacement velocity in disguise.

If disjoining pressure is positive at all film thicknesses, then as capillary number decreases, the thickness of the residual film tends to an asymptotic low value, which in the limit of vanishing capillary number depends on the width of the gap between the plates. In figure 5 results are shown for case 1 in table II, a hydrocarbon film on quartz in the presence of vapor - a case for which disjoining pressure is dominated by the molecular component, at least for films thicker than about five to ten molecules. In the limit of vanishing capillary number the capillary pressure difference across the meniscus in region 3 must be the same as the disjoining pressure in the residual film region 1 (cf. (16)). The slot width determines the curvature of the meniscus near the center of the slot and hence sets the residual film thickness. For the cases shown in figure 5, in a slot $0.2 \mathrm{~nm}$ wide the deposited film is $20 \mathrm{~nm}$ thick ; in a $2 \mu \mathrm{m}$ slot, it is only $3 \mathrm{~nm}$ thick. Likewise, according to equation (17), increasing the characteristic length for disjoining interactions thickens the film at fixed gap width.

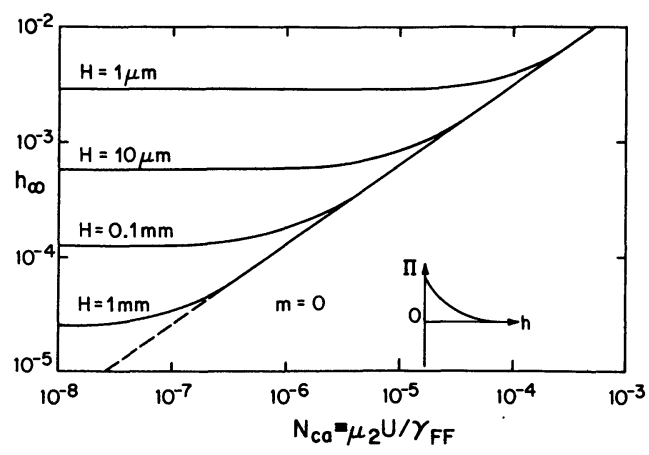

Fig. 5. - Dependence of a dimensionless film thickness on capillary number and gap width; wetting fluid.
On the other hand, when disjoining pressure is conjoining, i.e. $\partial \Pi / \partial h>0$ at all film thicknesses except perhaps for an adsorbed monolayer or so, there is a displacement rate below which no continuous film can be deposited, because the capillary pressure and viscous stresses are incapable of preventing conjoining collapse, that is, catastrophic thinning all along the apparent contact line the meniscus makes with the walls. To illustrate this phenomenon, a disjoining pressure function has been chosen that is appropriate to a vapor film sandwiched between the solid and a liquid hydrocarbon or to a hydrocarbon film trapped between the solid and water. In either of these cases, disjoining pressure is dominated by the molecular component, as shown in table II, case 2 . Results for very large viscosity ratio $m$, corresponding to the displacement of vapor by liquid, are shown in figure 6 . The capillary number in figure 6 is based on the viscosity of the vapor, the displaced phase. The residual film thins with decreasing displacement rate and eventually reaches a critical thickness below which the conjoining pressure gradient tending to thin the film overwhelms the viscous stresses tending to thicken it. For the example shown here the critical thickness for conjoining collapse is $10 \mathrm{~nm}$ in a $1 \mathrm{~mm}$ slot. The capillary number at which the critical film thickness occurs increases with decreasing gap width, or increasing conjoining interaction length (cf. Eq. (17)). Because $\partial \Pi / \partial h>0$ for all $h$, the thicker films which can in principle be deposited are unstable to disproportionation into patches and droplets surrounded by adsorbed monolayer (Goldsmith and Mason, 1962 ; Mohanty, 1981).

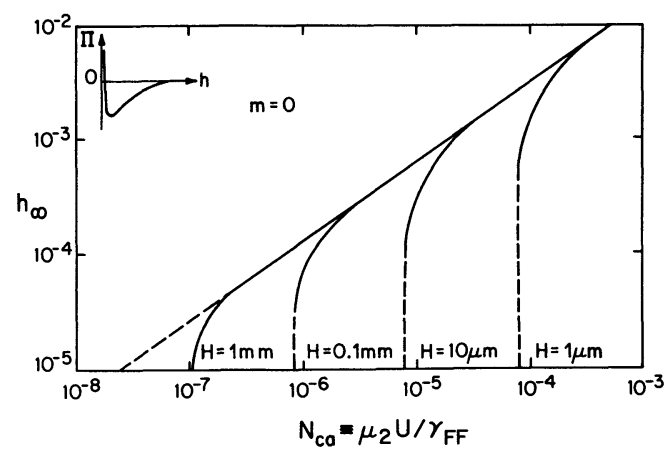

Fig. 6. - Dependence of dimensionless film thickness on capillary number and gap width; nonwetting fluid.

At any given capillary number, film thickness increases as viscosity ratio $m=\mu_{1} / \mu_{2}$ increases, as is shown in figure 7 for $N_{\mathrm{Ca}}=10^{-3}$, a capillary number for which disjoining pressure effects are negligible. In the limit $m \rightarrow \infty$, a rigid interface, film thickness tends to a value which is a factor of $2^{2 / 3}$ greater than the value for the limit $m \rightarrow 0$. The 
Table II. - Disjoining pressure functions.

System

Disjoining

Interfacial

pressure function, $\Pi(h)$

tension $\gamma_{\mathrm{FF}}$

1) Quartz-hydrocarbon-vapor

$A_{3} h^{-3}, A_{3}=1.0 \times 10^{-21}$ Joules

$30 \mathrm{mN} / \mathrm{m}$

2) Quartz-vapor-hydrocarbon or

$A_{3} h^{-3}, A_{3}=-1.0 \times 10^{-21}$ Joules

$30 \mathrm{mN} / \mathrm{m}$

Quartz-hydrocarbon-water

3) Quartz-water-vapor

$$
\begin{aligned}
& A_{1} / h, A_{1}=60 \mathrm{mN} / \mathrm{m} \quad h<80 \mathrm{~nm} \\
& A_{2} / h^{2}, A_{2}=2 \times 10^{-12} \mathrm{~N}, \quad h>120 \\
& \frac{\left(3 A_{1}+2 A_{2} / h_{1}\right)}{h}-\frac{2 A_{2}}{h^{2}}-\frac{2 A_{1}}{h_{1}} \\
& 80 \mathrm{~nm}<h<120 \mathrm{~nm} \quad h_{1}=80 \mathrm{~nm}
\end{aligned}
$$

$72 \mathrm{mN} / \mathrm{m}$

4) Solid-water-fluid

$A_{3} / h^{3}+E \mathrm{e}^{-\chi h}$

$30 \mathrm{mN} / \mathrm{m}$

$\psi_{1}=\psi_{2}=-75 \mathrm{mV}$

$\rho_{\mathrm{i}}=10^{-3} \mathrm{~mol} / 1$

$A_{3}=+1.0 \times 10^{-21}$ Joules

$E=+1.75 \times 10^{5} \mathrm{~N} / \mathrm{m}^{2}$

$\chi=10^{6} \mathrm{~cm}^{-1}$

5) Solid-water-fluid

$$
\begin{aligned}
& A_{3} / h^{3}+E \mathrm{e}^{-\chi h} \\
& A_{3}=-1.0 \times 10^{-21} \mathrm{Joules} \\
& E=-1.75 \times 10^{5} \mathrm{~N} / \mathrm{m}^{2} \\
& \chi=10^{6} \mathrm{~cm}^{-1}
\end{aligned}
$$

6) Solid-water-fluid

$$
\begin{aligned}
& A_{3} / h^{3}+E \mathrm{e}^{-\chi h} \\
& A_{3}=+1.0 \times 10^{-21} \mathrm{Joules} \\
& E=-1.75 \times 10^{5} \mathrm{~N} / \mathrm{m}^{2} \\
& \chi=10^{6} \mathrm{~cm}^{-1}
\end{aligned}
$$

$$
\begin{aligned}
& A_{3} / h^{3}+E \mathrm{e}^{-\chi h} \\
& A_{3}=-1.0 \times 10^{-21} \mathrm{Joules} \\
& E=+1.75 \times 10^{5} \mathrm{~N} / \mathrm{m}^{2} \\
& \chi=10^{6} \mathrm{~cm}^{-1}
\end{aligned}
$$$$
30 \mathrm{mN} / \mathrm{m}
$$

7) Solid-water-fluid or

vapor-water-vapor

8) Partially wetting liquid

$$
\begin{aligned}
& A_{3} / h^{3}-A_{2} / h^{2} \\
& A_{3}=1.0 \times 10^{-21} \text { Joules } \\
& A_{2}=2.0 \times 10^{-12} \mathrm{~N}
\end{aligned}
$$

flux of the film fluid in the $x$-direction, $q_{2}$, is a factor of $2^{-1 / 3}$ less than in the $m \rightarrow \infty$ limit than in the $m \rightarrow 0$ limit, however. These results for the $m \rightarrow \infty$ limit when disjoining pressure is negligible were previously elucidated by Bretherton (1961). Because the film thickness increases with increasing viscosity ratio, the critical capillary number for conjoining collapse decreases as viscosity ratio increases, as shown in figure 8.
The preceding examples are for combinations of non-polar materials having disjoining pressure functions dominated by the molecular contribution. But when ionic or polar species are present in the film the ionic-electrostatic and structural contributions are most significant. These contributions to disjoining pressure - and hence the thickness of the residual film - depend strongly on electrolyte concentration and interfacial charge. 


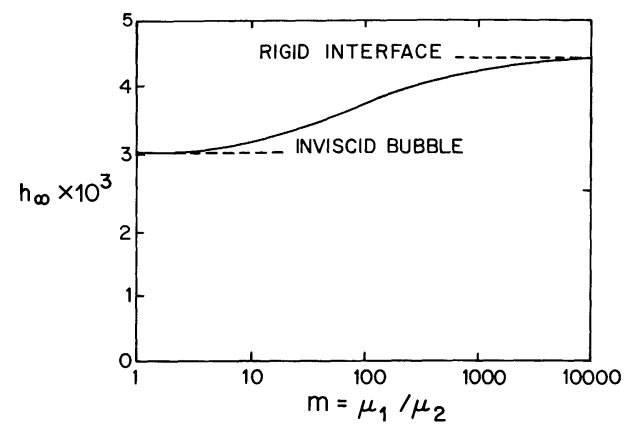

Fig. 7. - Dimensionless film thickness versus viscosity ratio for $N_{\mathrm{Ca}}=10^{-4}$.

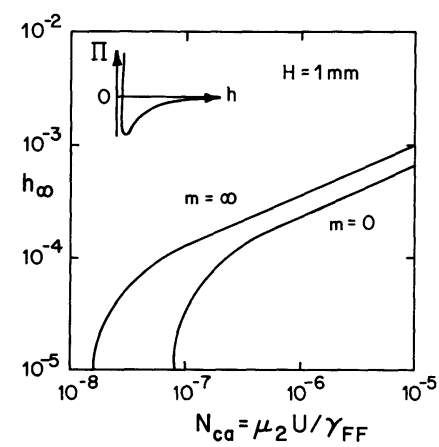

Fig. 8. - Effect of viscosity ratio on critical capillary number for conjoining collapse.

The disjoining pressure function for pure water on carefully cleaned silica surfaces is given in table II, case 3 . The corresponding film thickness vs. capillary number resembles that shown in figure 5. Because of the abrupt change in the disjoining pressure function near $100 \mathrm{~nm}$, the film thickness in the $N_{\mathrm{Ca}} \rightarrow 0$ limit approaches an asymptotic value which is nearly independent of gap width (Mohanty, 1981).

The pattern of film deposition can be changed dramatically by altering electrolyte concentration or interfacial charge when both the ionic-electrostatic and molecular or structural contributions to disjoining pressure are important. Four representative cases 4-7 are listed in table II. When $\Pi_{\mathrm{e}}$ and $\Pi_{\mathrm{m}}$ are both positive, case 4 , the variation of film thickness with capillary number is qualitatively similar to that shown in figure 7 , except that $\Pi_{\mathrm{e}}$ makes the residual film somewhat thicker than when $\Pi_{\mathrm{m}}$ alone is present. On the other hand, when both $\Pi_{\mathrm{e}}$ and $\Pi_{\mathrm{m}}$ are negative, case 5, the variation of film thickness with capillary number is similar to that shown in figure 6 except that conjoining collapse occurs when the residual film is somewhat thicker and the capillary number larger than when $\Pi_{\mathrm{m}}$ alone is present.

When the disjoining pressure function has both positive and negative regions, dynamic wetting behavior depends strongly on the ratios of molecular interaction lengths to gap width or tube radius. Large negative $\Pi_{\mathrm{e}}$, as in case 6 , can cause a film having positive $\Pi_{\mathrm{m}}$ to undergo conjoining collapse below a critical displacement speed if the capillary pressure jump in region 3 is small compared to the minimum of the disjoining pressure function, as shown in figure 9 . Conversely, large positive $\Pi_{\mathrm{e}}$ as in case 7 can prevent disjoining collapse if the capillary pressure jump in region 3 is small compared to the maximum of the disjoining pressure function, as shown in figure 10. In both cases 6 and 7 the effect of $\Pi_{\mathrm{e}}$ on wetting behavior is insignificant in sufficiently small slots or tubes, or at sufficiently high electrolyte concentration (cf. Eqs. (28-31)).

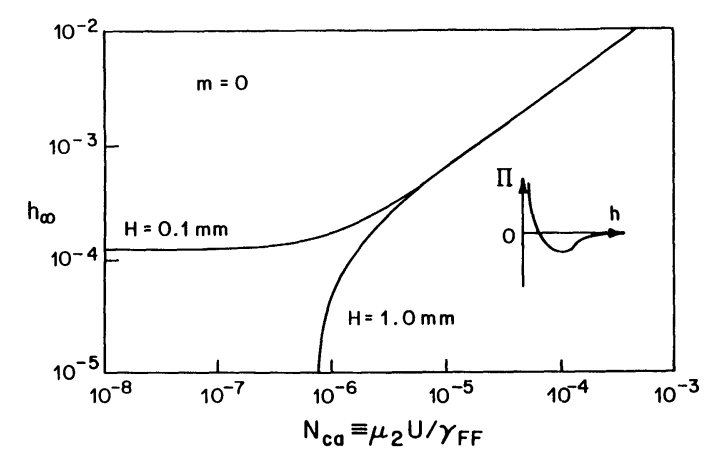

Fig. 9. - Dependence of dimensionless film thickness on capillary number and gap width, case 6 in table II.

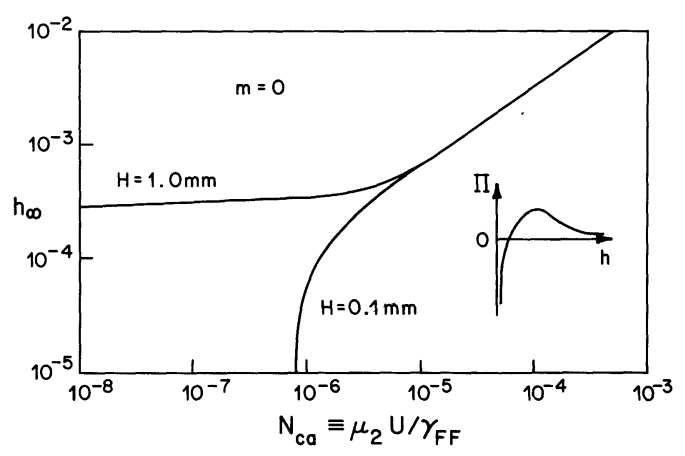

Fig. 10. - Dependence of dimensionless film thickness on capillary number and gap width, case 7 in table II.

The length scale on which disjoining pressure is significant is typically less than the wavelength of light. Thus even when one of the fluids completely wets the solid in a thin-film state, the meniscus at observable distances from the solid may appear to intersect the solid at a non-zero angle (Fig. 3). The contact angle that is seen either by naked eye or through an optical microscope is called the apparent contact angle and is, under conditions that are presumed to give equilibrium, what has long been called the equilibrium contact angle. The contact angles seen in non-equilibrium conditions are called 
the recently advanced, recent receded, or dynamic apparent contact angle (cf. Huh and Scriven, 1971).

If the meniscus far from the solid is uniformly curved, as when the meniscus is at rest or moving at $N_{\mathrm{Ca}}<10^{-2}$, the apparent contact angle that is measured is the angle at which the extrapolation of such a static meniscus intersects the solid surface. In particular, the apparent contact angle $\theta_{\mathrm{a}}$ of a cylindrical meniscus between parallel plates is given by

$$
\cos \theta_{\mathrm{a}}=H_{\kappa}
$$

where $H$ is half the gap width and $\kappa$ is the curvature of the static meniscus.

When $\Pi(h)>0$ for all thicknesses, then $1 / \kappa<H$ regardless of displacement speed. Thus the extrapolated interface does not intersect the solid surface, but the eye sees a zero contact angle if the residual film is thin compared to the gap width. In contrast, if $\Pi(h)<0$ for some thicknesses, e.g., case 8 in table II, then $1 / \kappa>H$ at low displacement speeds but $1 / \kappa<H$ at high displacement speeds. As a consequence, the eye sees a nonzero contact angle at low speeds but sees a zero contact angle (measured through the displaced phase) at high speeds. The velocity dependence of the apparent contact angle for case 8 is shown in figure 11. If the solid is smooth, the contact angle is predicted to retain its static value until the displacement rate is increased above a critical value beyond which the contact angle decreases rapidly with displacement rate, eventually reaching zero when the film becomes thick enough for disjoining pressure to be negligible.

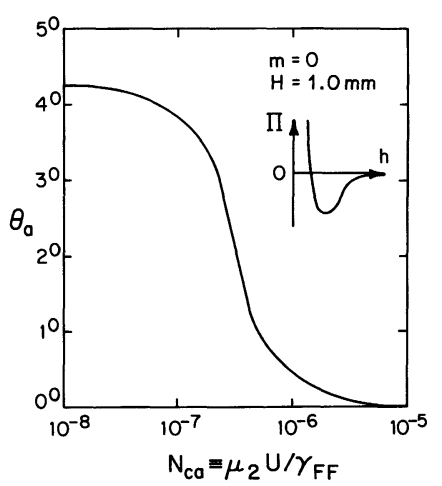

Fig. 11. - Dependence of apparent contact angle on capillary number, case 8 in table II.

When the dynamic apparent contact angle is too large, the film profile in the transition region between the virtually static meniscus and the uniform residual film may be appreciably inclined and curved. When this happens, the small-slope approximation leading to the film profile equation fails, and numerical integration yields a profile which does not have uniform curvature near the center of the slot.
Calculations show that the small-slope approximation fails when the apparent contact angle is larger than about $5^{\circ}$. In such cases, the film profile must be found by solution of the full Navier-Stokes system for two-dimensional steady flow, augmented by microstructural fluid forces. However, because the film profile equation reduces to the exact augmented Young-Laplace equation in the $N_{\mathrm{Ca}} \rightarrow 0$ limit and to the Landau-Levich equation when the residual film is thick enough for disjoining pressure to be negligible, the present analysis is useful on the one hand for predicting the displacement speed up to which the contact angle has its static value, and on the other hand for determining the displacement speed above which the contact angle is zero.

\section{Discussion.}

The results distinguish two regimes of capillary number for steady two-dimensional displacements in narrow slots and small-bore tubes having smooth, clean walls. At low capillary number, the displacement can be classified as quasi-static. In this regime, the film profile is governed almost entirely by capillarity and disjoining pressure : meniscus shape is given to a very good approximation by the augmented Young-Laplace equation. Residual film thickness and apparent contact angle have essentially the same values they would have were the meniscus motionless. At higher capillary number, wetting properties, as determined by disjoining pressure, are insignificant, and Bretherton's (1961) purely hydrodynamic theory governs the shape of the film. The transition between the two regimes is abrupt, extending over no more than one order of magnitude of capillary number. At sufficiently high capillary number, steady, two-dimensional flow would always deposit a film of displaced fluid on the solid surface and the contact angle would tend to zero regardless of the wetting properties of the materials in question under the conditions.

The residual film can break up into droplets, however (Mohanty, Davis and Scriven, 1982, Jain et al., 1979). The condition for stability with respect to infinitesimal shape fluctuations of a uniform film on a horizontal plate is (Frenkel, 1946 ; Mohanty, Davis and Scriven, 1982)

$$
\left(\frac{\partial \Pi}{\partial h}\right)_{h=h_{\infty}}<0,
$$

A residual film in a tube is unstable if $h>h_{\text {crit }}$, where $h_{\text {crit }}$ is given by (Mohanty, Davis and Scriven, 1982 ; Mohanty, 1981).

$$
\left[\sigma_{\mathrm{FF}}+R \Pi\left(h_{\text {crit }}\right)\right] / H+(\partial \Pi / \partial h) h_{\text {crit }}=0 .
$$

The characteristic time for breakup of the film is on the order of seconds or minutes if only the molecular component of conjoining pressure is present with 
$A_{3}=10^{-20}$ Joule and $10 \mathrm{~nm}<h_{\infty}<100 \mathrm{~nm}$ (Jain et al. 1979). If $A_{3}$ is larger or the film thickness smaller, the film ruptures within a small fraction of a second. In such cases, not only the residual film, but also the two-dimensional steady flow in the vicinity of the moving contact line (region 2) may be unstable to a three-dimensional unsteady flow and the fluid may be left behind as rivulets rather than as a uniform film.

Laboratory investigations support the qualitative, and often the quantitative predictions of the present analysis. Of the many experimental measurements of residual film thickness in tubes, only a few extend to the range of very small capillary number where disjoining, or conjoining, pressure is important. Bretherton (1961) experimented with the displacement by air or saturated vapor of benzene or aniline from a $1 \mathrm{~mm}$ diameter glass capillary. He found good agreement between his theory and measurements at capillary number between $10^{-5}$ and $10^{-2}$. But at capillary numbers less than $10^{-5}$, the measured film thickness was larger than he predicted, and at capillary number less than $10^{-6}$ the film thickness appeared to be independent of capillary number. Calculations with a realistic model of the disjoining pressure of pure benzene on clean glass (Fig. 5) predict a quasi-static film thickness of $25 \mathrm{~nm}$ in a $1 \mathrm{~mm}$ tube whereas Bretherton's indirect measurements give a film thickness of around $500 \mathrm{~nm}$. However, when only the molecular component of disjoining pressure is present any physically reasonable value of the constant $A_{3}$ leads to an equilibrium film thickness less than $100 \mathrm{~nm}$. Even with ionic impurities which can cause a significant ionic-electrostatic contribution to disjoining pressure, the equilibrium film thickness predicted for a $1 \mathrm{~mm}$ tube is less than $200 \mathrm{~nm}$.

Roughness of the tube walls could increase the amount of fluid left behind and cause the flow to become three-dimensional and unsteady. At equilibrium, both the characteristic height of the roughness and the characteristic distance between crests and valleys would have to be close to $1 \mu \mathrm{m}$ to account for the apparent film thicknesses observed by Bretherton (cf. Mohanty, 1981 ; Philip, 1978). Roughness of this magnitude can safely be assumed not to be present in the capillaries used by Bretherton.

The most likely cause for the discrepancy between theory and Bretherton's experiments is the presence of impurities. Bretherton and others (Schwartz et al., 1986) have reasoned that surface-active impurities, if present, would be convected from the nose of the bubble to its rear, thus causing the surfactant concentration to be lower at the nose than at the rear. As a result, the interfacial tension in region 3 would be greater than that in region 1 , causing an interfacial tension gradient in region 2 , which in turn would cause an apparent hardening of the free surface (Levich, 1962). The most stringent surfacechemical standards of purity and cleanliness, such as those described by Mysels and Florence (1970), are necessary to eliminate the apparent rigidity of the interface of gas bubbles rising freely in liquid.

If surface active contaminants are present in sufficient quantities to cause the liquid-vapor interface to be truly rigid, the no-slip condition applies at the interface and the displacement corresponds to viscosity ratio $m=\infty$ rather than $m=0$. Thus, even if disjoining pressure is negligible, the observed film thickness is a factor of $2^{2 / 3}$ thicker than predicted by Bretherton. However, Bretherton actually measured the amount of fluid left behind on a given length $l$ of tube. As noted by Bretherton, this quantity is a factor of $2^{1 / 3}$ smaller in the $m=\infty$ case than in the $m=0$ case. Thus, rigidity of the interface caused by the interfacial tension in region 3 being greater than that in region 1 cannot account for the observations.

However, a careful consideration of the flow at the nose of the bubble reveals that it is more likely that impurities cause the interfacial tension in region 3 to be less than that in region 1 (Herbolzheimer 1987). Because only a small amount of the liquid ahead of the bubble can flow past the bubble in the deposited film, almost all of the liquid flows back upstream along the center of the tube. Consequently, in the absence of surfactant, there is a stagnation point on the interface somewhere in region 3 (Fig. 12a). In the presence of a surface active impurity, the reverse flow at the nose causes a surface concentration gradient such that the surface concentration is a maximum at the tip of the bubble and a minimum somewhere in the transition region, region 2. Hence, an interfacial tension gradient is established with the interfacial tension in region 3 less than that in region 1 . The appropriate film profile equation governing this situation is (Teletzke, 1983 ; Teletzke, Davis and Scriven, 1987)

$$
\begin{aligned}
& \frac{\partial}{\partial x}(2 \pi)+\frac{H^{2}}{\gamma_{\mathrm{FF}}} \frac{\partial \Pi}{\partial h} h_{x}= \\
& =3 N_{\mathrm{Ca}} \frac{h-q}{h^{3}}+\frac{3}{2} \frac{H \mathrm{~d} \gamma_{\mathrm{FF}} / \mathrm{d} x}{\gamma_{\mathrm{FF}}} \frac{1}{h},
\end{aligned}
$$
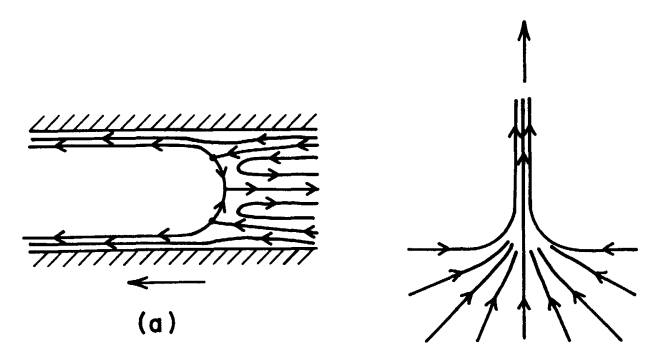

Fig. 12. - Sketches of streamlines : (a) bubble flow in a tube, showing stagnation points ; (b) pulling of a soap film from a bath, showing absence of stagnation points. 
where $\mathrm{d} \gamma_{\mathrm{FF}} / \mathrm{d} x$ is the surface tension gradient and $q \equiv q_{2} / U \mu$ is the dimensionless flux of fluid in the $x$ direction. Solutions of this equation by the procedure described above indicate that a difference in surface tension of only $0.1 \%$ between regions 1 and 3 is sufficient to account for Bretherton's observations. Therefore, the presence of impurities which cause the interfacial tension in region 1 to be greater than that in region 3 can be regarded as the most likely cause for the discrepancies between Bretherton's theory and experiment.

Further support for this interpretation is provided by measurements of film thickness obtained in dipcoating (Levich, 1942) and during the drawing of soap-films from liquid boths (Lyklema et al., 1965). As noted above, the film thicknesses in these experiments are governed by equations analogous to Bretherton's. However, in contrast to Bretherton's measurements, the film thicknesses determined in these experiments are in good agreement with theory, except for deviations which are clearly caused by disjoining or conjoining pressure (see below). A possible reason for the difference may be that the reverse flow and its associated surface concentration minimum do not occur during the withdrawal of films from baths (Fig. 12b).

Templeton $(1954,1956)$ and Goldsmith and Mason (1963) studied liquid-liquid displacements in tubes. Both found that the thickness of the residual film increases as the viscosity ratio $m=\mu_{1} / \mu_{2}$ increases when the capillary number $U \mu_{2} / \gamma_{\mathrm{FF}}$ (based on the viscosity of the displaced fluid) is held fixed. This result agrees with the present theory.

Elliott and Riddiford (1967) investigated dynamic contact angles during steady displacement between parallel plates, and Hoffman (1975) investigated the same during steady displacement in a capillary tube. Both studies were conducted at negligible Weber and Bond numbers. The meniscus at visually observable distances from the solid had constant curvature and the dynamic contact region appeared linear or circular and steady. The contact angle was measured by visually extrapolating the static meniscus to its intersection with the solid. Thus the results of these studies are directly comparable to the theory presented here.

In both studies, the solid was preferentially wet by the displacing fluid. Conjoining pressure functions are not presently known for the system studied by Elliott and Riddiford, viz. water displacing Bayol (a mixture of low molecular weight hydrocarbons) from between silicone-coated glass plates $1.5 \mathrm{~mm}$ apart nor for the system studied by Hoffman, silicone oil displacing air from a $2.0 \mathrm{~mm}$ glass capillary. However, the magnitudes of the capillary number $N_{\mathrm{Ca}, m}$ at which the contact angle begins to deviate from its static value, and of the capillary number $N_{\mathrm{Ca}, m}$ at which the contact angle reaches $0^{\circ}$, can be estimated by considering the magnitudes of the possible contributions to conjoining pressure. Because conjoining pressure is significant only in films less that $1 \mu \mathrm{m}$ thick, the maximum possible value of $N_{\mathrm{Ca}, m}$ is $10^{-5}$ for a $2 \mathrm{~mm}$ tube (cf. Fig. 6). $N_{\mathrm{Ca}, m}$ is the value of $N_{\mathrm{Ca}}$ at which viscous shear is negligible - less than $10^{-7}$ for a $2 \mathrm{~mm}$ tube (cf. Fig. 6). Both $N_{\mathrm{Ca}, m}$ and $N_{\mathrm{Ca}, M}$ decrease as the viscosity ratio $m$ increases (cf. Fig. 8). The values of $N_{\mathrm{Ca}, m}$ and $N_{\mathrm{Ca}, M}$ estimated from Elliott and Riddiford's and Hoffman's data are summarized in table III. In every case, the data are within the plausible ranges of $N_{\mathrm{Ca}, m}$ and $N_{\mathrm{Ca}, M}$ estimated from the magnitudes of the contributions to conjoining pressure.

Table III. - Values of $N_{\mathrm{Ca}, m}$ and $N_{\mathrm{Ca}, M}$ estimated from experiments.

\begin{tabular}{l|c|r|c}
\hline \multicolumn{1}{c|}{ Study } & $\begin{array}{c}\text { Viscosity } \\
\text { ratio } \\
m\end{array}$ & $N_{\mathrm{Ca}, m}\left(^{*}\right)$ & $N_{\mathrm{Ca}, M}\left(^{*}\right)$ \\
\hline $\begin{array}{l}\text { Elliott and Riddiford } \\
\text { (liquid displacing) }\end{array}$ & $10^{-2}$ & $<10^{-8}$ & $10^{-6}$ \\
Hoffman & $10^{5}$ & $10^{-8}$ & $10^{-5}$ \\
(liquid displacing air) & $10^{6}$ & $10^{-8}$ & $10^{-6}$ \\
& $10^{7}$ & $10^{-9}$ & $10^{-7}$ \\
& $10^{8}$ & $<10^{-9}$ & $10^{-8}$ \\
\hline
\end{tabular}

(*) $N_{\mathrm{Ca}} \equiv \mu_{2} U / \gamma_{\mathrm{FF}}$, where $\mu_{2}=$ viscosity of displaced fluid.

Several investigators examined the conditions of incipient air entrainment by tapes or fibers entering a bath of liquid (Inverarity, 1969; Burley and Kennedy, 1976 ; Blake and Ruschak, 1979 ; Gutoff and Kendrick, 1982). Entrainment becomes visually obvious when the capillary number based on the viscosity of the entrained fluid exceeds a critical value which falls in the range of $10^{-5}-10^{-3}$, which is rather higher than the plausible range of $N_{\mathrm{Ca}, M}$. Moreover, the flow is highly three-dimensional and unsteady when massive air entrainment begins. Fluid inertia is significant and gravity too in some circumstances. There is, however, evidence that true entrainment begins at lower speeds (Scriven 1982) that probably correspond closely to $N_{\mathrm{Ca}, M}$. But because the thin-film of air is unstable to conjoining pressure-driven instability that can break it down into adhering micro-bubbles, entrainment remains undetected until the volume of the entrained film becomes high enough to produce visible bubbles, or the film becomes thick enough to suffer threedimensional shear and inertial instabilities that break it up. The mechanisms are treated by Miyamoto and Scriven (1982).

The thickness of soap films pulled vertically from 
bulk solution are in close agreement with the theory presented here (Lyklema et al. 1965). For thickness greater than $50-100 \mathrm{~nm}$, good agreement with the Landau-Levich equation (called Frankel's law in that context) is found, but in thinner films, deviations occur. At low ionic strengths in the aqueous solution, electrostatic double-layer repulsion between the ionized film surfaces predominates and causes deviations toward larger thicknesses. At higher ionic strengths the double layers are thinner, and attractive Van der Waals forces become important, causing abrupt deviations toward thinner films stabilized by hydration forces. In all cases an equilibrium film thickness is reached at sufficiently low withdrawal speeds. These results are in agreement with the pattern of deposition behavior displayed in figure 10 .

Immiscible displacement in porous media is strongly affected by the wettability of the pore surfaces (see e.g. Craig, 1971). Calculations with a disjoining pressure function (including a doublelayer contribution) appropriate to a water film sandwiched between a mineral surface and a bulk layer of oil (case 6, Tab. II and Fig. 10) indicate that during the slow accumulation of crude oil into an initially water-filled pore space of a permeable rock, regions of the pore surface having curvature larger than a critical value set by the maximum in the disjoining pressure function are oil-wet and those having curvature less than the critical value are water-wet. This may be the origin of the so-called mixed-wettability states (Salathiel, 1973) of some petroleum reservoirs. Moreover, when a porous medium at equilibrium is completely wetted by the displacing fluid, but initially is completely filled by a less strongly wetting fluid (which of course covers the entire pore surface at the outset), it may appear to be wet by the displacing liquid at low capillary numbers but by the displaced fluid at higher capillary numbers : cf. figure 6 . On the other hand, if the pore surface is initially covered by a thin-film that is miscible with the displacing liquid, the porous medium appears to be wet by the latter at all capillary numbers.

This theory sheds light on the physics of flow in the submicroscopic contact region when the contact angle is not zero. At the submicroscopic level of the two-dimensional steady flow analyzed here, the meniscus does not truly intersect the solid surface, but joins with continuous slope to the residual film, or to a previously deposited film (Teletzke, 1983; Starov et al., 1977), or to a submicroscopic primary film advancing ahead of the meniscus (Teletzke, Davis and Scriven, 1987). Thus by accounting for microstructural fluid forces, the theory presented here provides an alternative to the Navier slip coefficient employed by some authors (Huh and Scriven, 1971 ; Huh and Mason, 1977 ; Silliman, 1979) and prescriptions of velocity discontinuity advocated by others (Dussan, 1979) to relieve the nonintegrably infinite stress singularity predicted by conventional macroscopic fluid mechanics at a contact line taken as a three-phase intersection.

In the calculations reported here, as in Templeton's $(1954,1956)$ experiments, the invisible residual film of displaced phase lubricates the flow of displacing fluid everywhere on the solid surface. As a consequence, if the existence of the residual film is overlooked or disregarded, the displacing phase can appear to slip, i.e. to have nonzero relative tangential velocity at the solid. When the residual film is unstable, as is generally the case when the dynamic apparent contact angle is not zero, the residual film retains its integrity at most a short distance beyond the apparent contact line. Then there is apparent slip only in the immediate vicinity of what the eye, whether naked or aided by a microscope, perceives as a contact line. The trapping of displaced fluid in surface roughness when present (Richardson, 1973 ; Hocking, 1976) and Knudsen flow in an entrained vapor film of thickness less than the mean free path (Miyamoto and Scriven, 1982) may also contribute to apparent slip at dynamic contact lines.

Elsewhere (Teletzke, 1983) we have calculated the profiles of short drops or bubbles by numerical integration of equation (16) in both the forward and backward directions, using a zeroth-order continuation procedure to step from longer to shorter bubbles. There it was found that the effective film thickness around a short bubble deviates from the film thickness predicted by Bretherton for long bubbles by less than $1 \%$ if the total length of the bubble exceeds about $\left(2+20 N_{\mathrm{Ca}}^{1 / 3}\right) R$. Moreover, other things being equal, shorter bubbles move faster than longer ones, and more viscous drops move faster than less viscous ones.

The low-speed steady displacements in horizontal tubes or slots considered here are prototypes for other displacements. The steady motion of drops or bubbles in vertical tubes (Bretherton, 1961), the entrainment of liquid during the steady withdrawal (or immersion) of solids out of (into) liquid baths (Khesghi and Scriven, 1979 ; Kistler and Scriven, 1979), and the drawing of soap films from liquid baths, (Mysels et al., 1959 ; Lyklema et al., 1965) have been similarly analysed in the small-slope approximation. The spontaneous spreading (Teletzke, Davis and Scriven, 1987) and rupture and retraction (Khesghi and Scriven, 1986) of liquid films on solids are unsteady flows which insofar as they are two-dimensional are governed in low-slope approximation by the time-dependent extension of equation (16).

The theory can be extended to the microfluid mechanics of thin-films and other fluid microstructures for which the small-slope approximation does not apply by substituting the appropriate approxi- 
mate velocity fields and expressions for the dependence of film tension on film thickness into the full integrodifferential momentum balance equation (Teletzke, 1983), or by solving the full NavierStokes system along with an equilibrium molecular theory for the pressure tensor. In this way threedimensional unsteady flows can be treated.

These approaches tacitly assume that bulk phase constitutive relations for viscous stress can be used to calculate the flux of fluid along the solid surface in the continuity equation. Though this approximation is surely valid for thick thin-films, it must break down as the film thickness approaches molecular dimensions. However, if as here, film thickness is taken to be proportional to the time-averaged number of molecules on the surface at a given location, the continuity equation is uniformly valid for all film thicknesses. In the limit of an adsorbed film corresponding to less than a monolayer, surface diffusion theory rather than continuum hydrodynamics can be used to relate fluxes to disjoining potential gradients and hence to film thickness gradients (Teletzke et al., 1987). The extension of the theory presented here to the microfluid mechanics of such thin thin-films, films having appreciable interfacial slope, multicomponent films in which interfacial mass transfer and interfacial tension gradients are significant and other fluid microstructures are important areas for future investigation.

\section{Acknowledgements.}

This research was supported by the Department of Energy, the National Science Foundation, and the University of Minnesota Computer Center.

\section{References}

[1] Blake, T. D. and RuschaK, K. J., A maximum speed of wetting, Nature 282 (1979) 489-491.

[2] Bretherton, F. P., The motion of long bubbles in tubes, J. Fluid Mech. 10 (1961) 166-188.

[3] (a) BuRley, R. and KENNEDY, B. S., A study of the dynamic wetting behavior of polyester tapes, $\mathrm{Br}$. Polym. J. 8 (1976) 140-143;

(b) Burley, R. and Kennedy, B. S., An experimental study of air entrainment at a solid/liquid/gas interface, Chem. Eng. Sci. 31 (1976) 901-911.

[4] Buscall, R. and Ottewill, R. H., Thin films, in Specialist Periodical Report, Colloid Sci. Ed. D. H. Everett (The Chemical Society, London) 2 (1975) 191-245.

[5] CASimir, H. G. B. and Polder, D., The influence of retardation on the London-Van der Waals forces, Phys. Rev. 73 (1948) 360.

[6] Craig, F. F., The Reservoir Engineering Aspects of Waterflooding (Society Petroleum Engineers, Dallas, Texas) 1971.

[7] DeBoer, J. H., The influence of Van der Waals' forces and primary bonds on binding energy, strength, and orientation, with special reference to some artificial resin, Trans. Faraday Soc. 32 (1936) $10-38$

[8] De Gennes, P. G., Wetting : Statics and Dynamics, Rev. Mod. Phys. 57 (1985) 827-863.

[9] Deryagin, B. V. and Landau, L. D., Theory of the stability of strongly charged lyophobic sols and of the adhesion of strongly charged particles in solutions of electrolytes, Acta Physicochim. 14 (1941) 633-662.

[10] Deryagin, B. V., The definition and magnitude of disjoining pressure and its role in the statics and dynamics of thin fluid films, Kolloid $\mathrm{Zh} .17$ (1955) 205-214.

[11] Deryagin, B. V., Correct form of the equation of capillary condensation in porous bodies, Proc. 2nd Int. Congr. Surf. Act. 2 (1957) 153-159.
[12] Deryagin, B. V. and ChuraeV, N. V., Structural component of disjoining pressure, J. Colloid Interf. Sci. 49 (1974) 249-255.

[13] DevereauX, O. F. and DE BrUYN, P. L., Interaction of Plane Parallel Double Layers (MIT Press, Cambridge, Mass) 1963.

[14] Dussan, V. E. B., On the spreading of liquids on solid surfaces : static and dynamic contact lines, Ann. rev. Fluid Mech. 11 (1979) 371-400.

[15] Dzyaloshinskit, I. E., LifShitz, E. M. and PITAEVSKII, L. P., Van der Waals forces in liquid films, Sov. Phys. JETP 37 (1960) 161-170.

[16] Elliot, G. E. P. and Riddiford, A. C., Dynamic contact angles. I. The effect of impressed motion, J. Colloid Interface Sci. 23 (1967) 389-398.

[17] Frenkel, J., Kinetic Theory of Liquids (Oxford University Press, Oxford) 1946.

[18] Goldsmith, H. L. and Mason, S. G., The flow of suspensions through tubes. II. Single large tubes, J. Colloid Sci. 18 (1963) 237-261.

[19] GutofF, E. B. and Kendrick, C. E., Dynamic contact angles, AIChE J. 28 (1982) 459-466.

[20] Hamaker, H. C., The London-Van der Waals attraction between spherical particles, Physica 4 (1937) 1058-1072.

[21] Herbolzheimer, E., The effect of surfactant on the motion of a bubble in a capillary, Paper \#209 presented at the ACS 61st Colloid Surf. Sci. Symp., Ann Arbor, MI (June 1987) to be submitted to J. Fluid Mech. (1987).

[22] Higgins, B. G. and SCRIVEN, L. E., Interfacial shape and evolution equations for liquid films and other viscocapillary flows, I \& EC Fund. 18 (1979) 208-215.

[23] Hindmarsh, A. C., GEAR : Ordinary differential equation system solver, Lawrence Livermore Laboratory Report UCID-30001, Revision 3 (1974).

[24] Hocking, L. M., A moving fluid interface on a rough surface, J. Fluid Mech. 76 (1976) 801-817. 
[25] Hogg, R., Healy, T. W. and Fuerstenau, D. W., Mutual coagulation of colloidal dispersions, Trans. Faraday Soc. 62 (1966) 1638-1651.

[26] Hoffman, R., A study of the advancing interface, I. Interface shape in liquid-gas systems, J. Colloid Interface Sci. 50 (1975) 228-241.

[27] HuH, C. and Scriven, L. E., Hydrodynamic model of steady movement of a solid/liquid/fluid contact line, J. Colloid Interface Sci. 35 (1971) 85-101.

[28] HuH, C. and MASON, S. G., The steady movement of a liquid meniscus in a capillary tube, J. Fluid Mech. 81 (1977) 401-419.

[29] InVERARITY, G., Dynamic wetting of glass fibre and polymer fibre, Br. Polym. J. 1 (1969) 245-251.

[30] IvanOV, I. B. and JAIN, R. K., Formation and thinning of liquid films, in Lect. Notes Phys., No. 105, Dynamics and Instability of Fluid Interfaces, Ed. T. S. Sorensen (Springer, Berlin) 1979.

[31] Jain, R. K., Ivanov, I. B., Maldarelli, C. and RUCKENSTEIN, E., Instability and rupture of thin liquid films, in Lect. Notes Phys., No 105, Dynamics and Instability of Fluid Interfaces, Ed. T. S. Sorensen (Springer, Berlin) 1979.

[32] JoANNY, J. F., Dynamics of wetting : Interface profile of a spreading liquid, J. Theor. Appl. Mech. Numero Special, (1986) pp. 249-271.

[33] JoAnNy, J. F. and Andelman, D., Steady-state motion of a solid-liquid-liquid contact line, $J$. Colloid Interface Sci. 119 (1987) 451.

[34] Kheshgi, H. S. and Scriven, L. E., Rising film flow : first-order approximation solved, Bull. Am. Phys. Soc. 24 (1979) 1131.

[35] Kheshgi, H. S. and SCRIVEN, L. E., Dewetting : nucleation and growth of dry regions, submitted to Chem. Eng. Sci. (1987).

[36] Kistler, S. F. and Scriven, L. E., Falling film flow : first-order approximation solved, Bull. Am. Phys. Soc. 24 (1979) 1131.

[37] Landau, L. D. and LeVich, V. G., Dragging of a liquid by a moving plate, Acta Physicochim. USSR 17 (1942) 42-54.

[38] LANGMUIR, I., The role of attractive and repulsive forces in the formation of tactoids, thixotropic gels, protein crystals, and coacervates, J. Chem. Phys. 6 (1938) 873-896.

[39] Levich, V. G., Physicochemical Hydrodynamics, (Prentice-Hall, Englewood Cliffs, N. J.) (1962).

[40] Lifshitz, E. M., The theory of molecular attractive forces between solids, Sov. Phys. JETP 2 (1956) 73-83.

[41] Lyklema, J., Scholten, P. L. and Mysels, K. J., Flow in thin liquid films, J. Phys. Chem. 69 (1965) 116-123.

[42] MahantY, J. and Ninham, B. W., Dispersion Forces (Academic Press, New York) 1976.

[43] Miyamoto, K. and Scriven, L. E., Breakdown of air film entrained by liquid coated on web, Paper $101 \mathrm{~g}$ presented at AIChE Annu. Meeting (Los Angeles, CA) Nov. 1982, to be submitted to AIChE J.

[44] Mohanty, K. K., Davis, H. T. and Scriven, L. E., Thin-films and fluid distributions in porous media, in Surface Phenomena in Enhanced Oil Recovery, Ed. D. O. Shah (Plenum Publ. Co., New York) (1981) pp. 395-609.

[45] Mohanty, K. K., Davis, H. T. and Scriven, L. E., Shape and stability of wetting fluid layers on simply shaped solids, AIChE Preprint 121a, presented at AIChE Annu. Meeting (San Francisco, CA) November 1979, Submitted to $J$. Colloid Interface Sci. (1988).

[46] Mohanty, K. K., Fluids in porous media : twophase distribution and flow, $\mathrm{Ph}$. D. Thesis, University of Minnesota (1981).

[47] Mysels, K. J., Shinoda, K. and Frankel, S., Soap films, Studies of Their Thinning, and a Bibliography (1959) Chapter V, pp. 47-65.

[48] Mysels, K. J. and Florence, A. T., Techniques and criteria in the purification of aqueous surfaces, in Clean Surfaces : Their Preparation and Characterization for Interfacial Studies, Ed. G. Goldfinger (Marcel Dekker, New York) 1970.

[49] Oliver, J. F., Huh, C. and Mason, S. G., Experimental study of some effects of solid surface roughness on wetting, Colloids Surf. 1 (1980) 79104.

[50] OHShima, H., Diffuse double layer interaction between two parallel plates with constant surface charge density in an electrolyte solution. I. Interaction between Similar Plates. II. Dissimilar plates, Colloid Polym. Sci. 252 (1974) 158-164, 257-267.

[51] PAShley, R. M., DLVO and hydration forces between mica surfaces in $\mathrm{Li}+, \mathrm{Na}+, \mathrm{K}+$, and $\mathrm{Cs}+$ electrolyte solutions : a correlation of doublelayer and hydration forces with surface cation exchange properties, J. Colloid Interface Sci. 84 (1981) 531-546.

[52] Pashley, R. M. and QUiRK, J. P., The effect of cation valency on DLVO and hydration forces between macroscopic sheets of muscovite mica in relation to clay swelling, Colloids Surf. of (1984) 1-17.

[53] PASHley, R. M., Multilayer adsorption of water on silica : an analysis of experimental results, $J$. Colloid Interface Sci. 78 (1980) 246-248.

[54] Parsegian, V. A. and Ninham, B. W., Application of the Lifshitz theory to the calculation of Van der Waals forces across thin liquid films, Nature 224 (1969) 1197-1198.

[55] Philip, J. R., Adsorption and capillary condensation on rough surfaces, J. Phys. Chem. 82 (1978) 1379-1385.

[56] RICHARDSON, S., On the no-slip boundary condition, J. Fluid Mech. 59 (1973) 707-719.

[57] SalathiEl, R. A., Oil recovery by surface film drainage in mixed-wettability rocks, J. Petr. Technol. 25 (1973) 1216-1224.

[58] Schwartz, L. W., Princen, H. M. and Kiss, A. D., On the motion of bubbles in capillary tubes, $J$. Fluid Mech. 1972 (1986) 259-275.

[59] SCRIVEN, L. E., How does air entrain at wetting lines ?, Paper 46a presented at AIChE National Meeting (Orlando, FL) March 1982, to be submitted to Chem. Eng. Commun. (1987). 
[60] Sheludko, A., Thin liquid films, Adv. Colloid Interf. Sci. 1 (1968) 391-464.

[61] Silliman, W. J., Viscous film flows with contact lines: finite element simulation, a basis for stability assessment and design optimization; Ph. D. Thesis, University of Minnesota (1979).

[62] Starov, V. M., Churaev, N. V., KhvorosTYAROV, A. G., The form of the moving meniscus in flat capillaries, Kolloid $Z h .39$ (1977) 201205.

[63] Templeton, C. C., A study of displacements in microscopic capillaries, Trans. AIME 201 (1954) 162-168.

[64] Templeton, C. C., Oil-water displacements in microscopic capillaries, 207 (1956) 211-214.

[65] Teletzke, G. F., Thin liquid films, molecular theory and hydrodynamic implications, Ph. D. Thesis, University of Minnesota (1983).

[66] Teletzke, G. F., Davis, H. T. and Scriven, L. E., How liquids spread on solids, AIChE Preprint 94f, presented at AIChE Annual Meeting, November 1981 (New Orleans) Chem. Eng. Commun. 55 (1987) 41-81.

[67] Teletzke, G. F., SCRiven, L. E. and Davis, H. T., Gradient theory of wetting transitions, J. Colloid Interface Sci. 87 (1982) 550-571.

[68] Vaillant, M. P., Sur un procédé de mesure des grandes résistances polarisables et son application à la mesure de la résistance de liquides, C.R. Hebd. Séan. Acad. Sci. Paris, 156 (1913) 307-310.

[69] Verwey, E. J. W. and Overbeek, J. Th. G., Theory of the Stability of Lyophillic Colloids (Elsevier, Amsterdam) 1948.

[70] VRu, A., Possible mechanisms for the spontaneous rupture of thin, free liquid films, Discurs. Faraday Soc. 42 (1966) 23-33.

[71] WeST, G. D., On resistance to the motion of a thread of mercury in a glass tube, Proc. R. Soc. Ser. A 86 (1911) $20-25$ 